\title{
Animal testing alternatives come alive in US
}

In Europe, long-standing public opposition toward animal testing has led to a broad push to develop alternative means for assessing the potential hazards of drugs. But similar efforts across the Atlantic have often lagged far behind. Now, with the formation of a new society dedicated to finding nonanimal testing methods, as well as new government programs, many experts perceive a sea change in US policy.

"There just seems to be an uprising and enthusiasm in the US for finding these alternative methods," says Erin Hill, vice president of program development at the Institute for In Vitro Sciences, a nonprofit testing center in Gaithersburg, Maryland.

Hill, together with Kristie Sullivan from the Physicians Committee for Responsible Medicine, unveiled the new American Society for Cellular and Computational Toxicology' at the In Vitro Alternatives Forum in Alexandria, Virginia this past October. Although only a handful of non-board members have signed up to the society thus far, "we're getting a couple more each day as word gets out," says Sullivan.

"It's an important step," remarks Thomas Hartung, director of the Center for Alternatives to Animal Testing at Johns Hopkins University in Baltimore and a member of the new society's board of directors. He points to a "long-lasting tradition in Europe of alternative methods" for testing compounds, adding that "in the US there was no equivalent" until now.

The formation of the new society comes three years after the US National Research Council (NRC) issued a report calling for a complete overhaul in the way chemicals, pharmaceuticals and pollutants are assessed for toxicity. To help implement the report, the Humane Society of the United States, the Hamner Institutes for Health Sciences and a number of key industrial stakeholders, including Dow Chemical, Johnson \& Johnson and Procter \& Gamble, teamed up last year to form the Human Toxicology Project Consortium. The consortium held its first public symposium last month in Washington, DC.

"We're trying to drum up a sense of buy-in and urgency to the NRC vision and how it might best be implemented," says Martin Stephens, the Humane Society's vice president of animal research issues.

Government agencies have heeded the call. In July, for instance, the US Food and Drug Administration (FDA) joined the Tox21 initiative, an interagency effort to develop models for more effective chemical risk assessments. The collaboration, which also

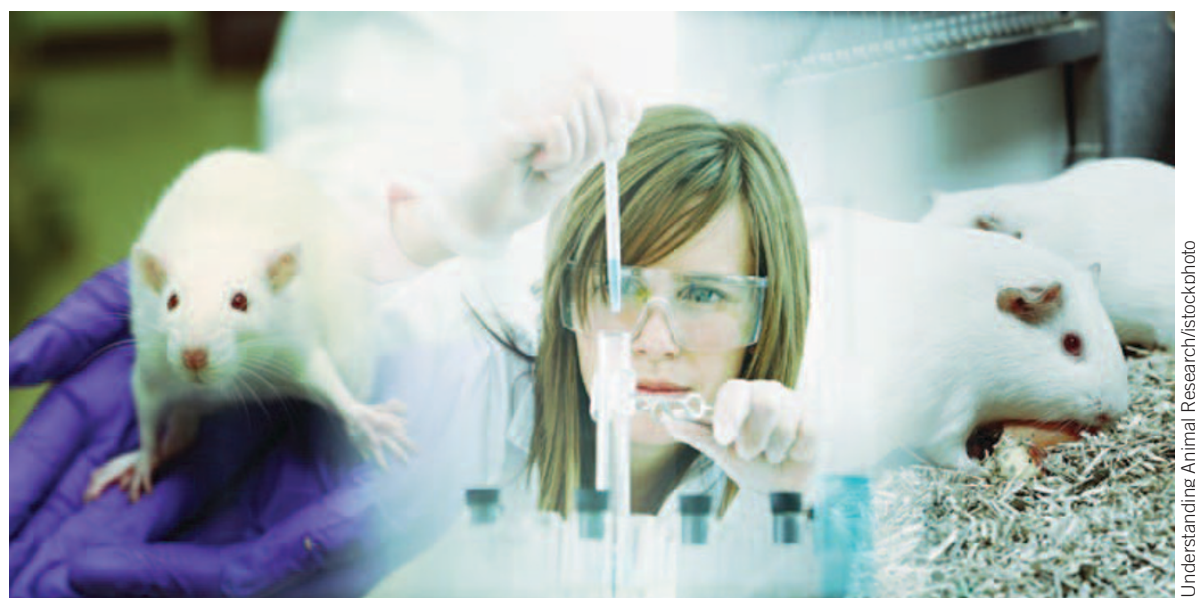

In vitro, in vogue: Lab dishes replace animals in toxicological testing.

involves the US Environmental Protection Agency (EPA) and the US National Institutes of Health, aims to determine an experimental compound's safety earlier in the drug discovery process by comparing its molecular characteristics to a database of around 3,000 pharmaceutical compounds and 7,000 environmental chemicals with known toxicity profiles. Next, the initiative plans to add watersoluble compounds and complex mixtures of chemicals, too.

"The goal is to develop fingerprints for compounds," explains Raymond Tice, chief of the Biomolecular Screening Branch at the US National Institute for Environmental Health Sciences' National Toxicology Program in Research Triangle Park, North Carolina. "The whole process we're going for is to try and link chemicals to genes, genes to pathways and pathways to disease."

\section{In vitro incentives}

This resource should prove to be a boon to the drug industry, says David Jacobson-Kram, the FDA's associate director for pharmacology and toxicology. "In the future, these tools will be available to pharmaceutical companies very early on in selecting their lead compounds to more effectively choose the chemical structures that can become drugs instead of failing because of unexpected toxicity."

The benefits of nonanimal testing are not lost on the pharmaceutical industry, notes Rodger Curren, president of the Institute for In Vitro Sciences, whose clients include many large drug manufacturers. "There's a significant drive to reduce animals," which helps in the court of public opinion, he says. "But there's equally a strong drive to get better information and to get it in an economically feasible fashion."
As part of Tox21, the EPA has also completed the first phase of its ToxCast program, which involved rigorously testing around 300 chemicals (mostly pesticides) in close to 500 assays in multiple human and animal cell lines to determine which chemicals activate different metabolic pathways (Environ. Health Perspect. 118, 485-492, 2010). Comparing the results with in vivo data, the researchers found that the more cellular pathways perturbed by a chemical as observed in a lab dish, the lower the dose at which the chemical causes toxicity in animals.

According to Robert Kavlock, director of the EPA's National Center for Computational Toxicology (NCCT) in Research Triangle Park, North Carolina, the agency now plans to add more assays and another 700 chemicals, including some failed drug candidates donated by pharma giants including Pfizer, Merck, GlaxoSmithKline and Sanofi-Aventis.

"This is probably the world's largest collection of consistently collected animal and in vitro data," says Kavlock, adding, "we can really use this information to target the use of animals more appropriately."

Melvin Andersen, director of chemical safety at the Hamner Institutes, applauds the NCCT's efforts but worries that Toxcast focuses more on "assays of convenience than assays designed for purpose." He argues that to really develop in vitro diagnostics of risk assessment-rather than just tests that raise red flags for hazardous chemicals-more discussions are needed into the best assays to probe specific metabolic pathways before high-throughput techniques are widely implemented. "We're getting the cart before the horse a little bit for risk assessment," he says.

Elie Dolgin 